\title{
Pairs of Normal Matrices With Property $L$
}

\author{
Helmut Wielandt ${ }^{1}$
}

\begin{abstract}
A short proof is given, under weaker assumptions, of the following theorem first proved by $\mathrm{N}$. Wiegmann: If the eigenvalues $\alpha_{i}, \beta_{i}$ of two normal $n \times n$ matrices $A, B$ may be numbered in such a way that the eigenvalues $\gamma_{i}(z)$ of $C(z)=a+z b$ are given by $\gamma_{i}(z)=\gamma_{i}+$ $z \beta_{i}$ (for $i=1, \ldots, n$ and all complex values of $z$ ), then $A B=B A$.
\end{abstract}

Two $n \times n$ matrices $A, B$ are said ${ }^{2}$ to have property $L$ if their eigenvalues $\alpha_{k}, \beta_{k}(k=1, \ldots, n)$ may be ordered in such a way that the eigenvalues of $\alpha A+\beta B$ are given by $\alpha \alpha_{k}+\beta \beta_{k}$ for all complex numbers $\alpha, \beta$. Though every pair of commuting matrices has property $L$, the converse is not true. ${ }^{2}$ However, if $A$ and $B$ have property $L$ and are normal, then $A B=B A$ according to a theorem of Wiegmann. ${ }^{3}$ We wish to show that the assumptions of Wiegmann's theorem may be weakened considerably. We begin by proving the following lemma which has some interest in itself.

Lemma: Let $A$ and $B$ be arbitrary $n \times n$ matrices. Then the set $Z$ of all points $z$ in the complex plane for which $A+z B$ is normal, is either the whole plane, a straight line, a circle, or it contains, at most, two points. If $B$ is normal, neither the circle case nor the two-point case may enter. If $Z$ is the entire plane then $A B=B A$.

Proof: $A+z B$ is normal if, and only if,

$$
(A+z B)\left(A^{*}+\bar{z} B^{*}\right)-\left(A^{*}+\bar{z} B^{*}\right)(A+z B)=0 .
$$

If $z=x+i y,(1)$ is equivalent to a set of $2 n^{2}$ real linear equations in $x, y$, and $\left(x^{2}+y^{2}\right)$. Each of these $2 n^{2}$ equations defines either the whole plane, a circle, a straight line, or else, at most, one point. The intersection of these $2 n^{2}$ point sets obviously is one of the six types described in the lemma. That all these types may indeed occur is shown by the following matrices $A+z B$ :

$$
\begin{gathered}
(1+z),\left(\begin{array}{ll}
1 & z \\
z & 0
\end{array}\right),\left(\begin{array}{ll}
0 & z \\
1 & 0
\end{array}\right), \\
\left(\begin{array}{lll}
0 & z & 0 \\
0 & 0 & z \\
z & 0 & z-1
\end{array}\right),\left(\begin{array}{ll}
0 & z \\
0 & 0
\end{array}\right),\left(\begin{array}{ll}
0 & 1 \\
0 & 0
\end{array}\right) .
\end{gathered}
$$

In case $B$ is normal, the terms of (1) containing $z \bar{z}$ cancel, hence $Z$ is determined by linear equations in $x$ and $y$ only. So $Z$ is neither a circle nor a pair of points.

\footnotetext{
1 American University and University of Tübingen. This paper was prepared under a National Bureau of Standards contract with The American University . ${ }_{2}$ T. S. Motzkin and O. Taussky, Pairs of matrices with property $L$, Trans. Am. Math. Soc. 73, 108-114 (1952)

3 N. Wiegmann, Pairs of normal matrices with property $L$, Proc. Am. Math. Soc. 4, 35-36 (1953).
}

In case $Z$ is the entire plane (1) holds for every $z$, hence the coefficient of $z$ vanishes, that is, $B A^{*}-A^{*} B=0$. Since $B$ commutes with $A^{*}$, it also commutes with every polynomial $f\left(A^{*}\right)$, hence, with $A$ itself. (If $A$ is normal, then $A=f\left(A^{*}\right)$ for some polynomial $f$. This well-known fact follows by transforming $A$ into diagonal form by means of a unitary transformation.) The lemma being proved, we turn to the generalization of Wiegmann's theorem.

Theorem: If the eigenvalues $\alpha_{k}$, $\beta_{k}$ of two normal matrices $A=\left(a_{i k}\right), B=\left(b_{i k}\right)$ may be ordered in such $a$ way that the eigenvalues $\gamma_{k}(z)$ of $A+z B$ satisfy the inequalities

$$
\sum_{k=1}^{n}\left|\gamma_{k}(z)\right|^{2} \geqq \sum_{k=1}^{n}\left|\alpha_{k}+z \beta_{k}\right|^{2}
$$

for some values $z=z_{\lambda}(\lambda=1, \ldots, l)$ which are the vertices of a polygon containing 0 in its interior, then $A B=B A$. (This obviously contains Wiegmann's theorem since if $A$ and $B$ have property $L$ then (2) holds even with equality sign for every $z$.)

Proof: According to a theorem of Schur, ${ }^{4}$ we have

$$
\sum_{i, k}\left|a_{i k}+z b_{i k}\right|^{2} \geqq \sum_{k}\left|\gamma_{k}(z)\right|^{2}
$$

for every $z$, where the equality holds if, and only if, $A+z B$ is normal. From (3) and (2) we have for $z=z_{\lambda}$

$$
\sum_{i, k}\left|a_{i k}+z b_{i k}\right|^{2} \geqq \sum_{k}\left|\alpha_{k}+z \beta_{k}\right|^{2}
$$

which reduces to

$$
\sum_{i, k}\left(a_{i k} \bar{z} \bar{b}_{i k}+\bar{a}_{i k} z b_{i k}\right)-\sum_{k}\left(\alpha_{k} \bar{z}_{\beta_{k}}+\bar{\alpha}_{k} z \beta_{k}\right) \geqq 0
$$

since $\sum\left|a_{i k}\right|^{2}=\sum\left|\alpha_{k}\right|^{2}$ and $\sum\left|b_{i k}\right|^{2}=\sum\left|\beta_{k}\right|^{2}$ in view of the fact that $A$ and $B$ are normal. Since the left-hand side of (5) is linear and homogeneous in $x$ and $y,(5)$ defines either a half plane containing 0 on its boundary or the left-hand side of (5) vanishes identically. The former case cannot occur, for the half plane contains $z_{1}, \ldots, z_{l}$, hence it contains 0 in its interior. So in (5), and hence in (4), equality holds for every $z$. Combining this with (2) and (3) we

${ }^{4}$ I. Schur, Über die charakterischen Wurzeln einer linearen Substitution mit einer Anwendung auf die Theorie der Integralgleichungen, Math. Ann. 66, 488-510 (1909); Theorem II. The theorem of Schur has recently been used in a related problem by $\mathrm{H}$. Schneider, Theorems on normal matrices, Quart. J. Math. Oxford [2] 3, 241-249 (1952). 
conclude that in (3) equality holds for $z=z_{\lambda}$, hence $A+z_{\lambda} B$ is normal. Since $z_{1}, \ldots, z_{2}$ are not collinear and $B$ is normal, the lemma asserts that $A+z B$ is normal for every $z$, hence $A B=B A$.

Remark: The theorem is best possible in the sense that there exist noncommuting normal matrices $A, B$ such that inequality (2) holds for every $z$ of a closed half plane containing 0 on its boundary (and for a fixed suitable ordering of the eigenvalues $\alpha_{k}, \beta_{k}$ ). An example is given by

$$
A=\left(\begin{array}{rrrr}
1 & 0 & 0 & 0 \\
0 & -1 & 0 & 0 \\
0 & 0 & i & 0 \\
0 & 0 & 0 & -i
\end{array}\right), \quad B=\left(\begin{array}{rrrr}
0 & 0 & 0 & 0 \\
0 & 0 & 0 & 0 \\
0 & 0 & 0 & 1 \\
0 & 0 & -1 & 0
\end{array}\right)
$$

with the ordering $\alpha_{k}=1,-1, i,-i ; \beta_{k}=i,-i, 0,0$. Here we have $\gamma_{k}(z)=1,-1,\left(-1-z^{2}\right)^{\frac{1}{2}},-\left(-1-z^{2}\right)^{\frac{1}{2}}$, hence $\sum\left|\gamma_{k}(z)\right|^{2} \geqq \sum\left|\alpha_{k}+z \beta_{k}\right|^{2}$ for every $z$ with $y \geqq 0$ (though $\gamma_{k}(z)=\alpha_{k}+z \beta_{k}, k=1, \ldots, 4$ is valid for $z=0$ only).

Washington, April 17, 1953. 